\title{
Philosophiques
}

\section{La notion d'authenticité chez Husserl et Heidegger}

\section{R. Philip Buckley}

Volume 20, numéro 2, automne 1993

Perspectives sur la phénoménologie et l'intentionnalité

URI : https://id.erudit.org/iderudit/027233ar

DOI : https://doi.org/10.7202/027233ar

Aller au sommaire du numéro

Éditeur(s)

Société de philosophie du Québec

ISSN

0316-2923 (imprimé)

1492-1391 (numérique)

Découvrir la revue

Citer cet article

Buckley, R. P. (1993). La notion d'authenticité chez Husserl et Heidegger.

Philosophiques, 20(2), 399-422. https://doi.org/10.7202/027233ar d'utilisation que vous pouvez consulter en ligne.

https://apropos.erudit.org/fr/usagers/politique-dutilisation/ 


\section{LA NOTION D’AUTHENIICIIÉ CHEZ HUSSERL ET HEIDECGER}

\section{par \\ R. Philip Buckley}

D'entrée de jeu, la « neutralité morale » pourrait sembler caractériser la phénoménologie. Car, après tout, la phénoménologie fut initialement conçue comme « dénuée de toutes présuppositions » ou «purement descriptive », sa tâche étant de rendre compte adéquatement de la structure intentionnelle de la conscience. Malgré la transformation apportée par Heidegger au projet original de Husserl pour en faire une ontologie phénoménologique, ses premiers pas vers une « analyse » du « comportement » intentionnel du Dasein ont l'apparence d'une entreprise « formelle » similaire qui, d'aucune façon, ne plaiderait en faveur d'un type de « comportement » sur un autre. Néanmoins, il demeure difficile d'aborder tant les textes de Husserl que ceux de Heidegger sans avoir à tout le moins une « impression » que quelque chose de propre à la prescription est à l'œuvre. Cela est certainement le cas lorsque ces auteurs traitent de l'« authenticité » et de l' « inauthenticité ». Je me propose non seulement de clarifier pourquoi cette « impression » de prescription peut advenir (et ainsi suggérer quelques points de convergence surprenants entre Husserl et Heidegger), mais également d'indiquer les énormes enjeux impliqués dans toutes discussions portant sur la vie humaine authentique.

\section{Qu'est-ce que l'authenticité pour Husserl?}

Quoique les notions d'authenticité et d'inauthenticité soient généralement associées à Heidegger et à Sartre, cette distinction joue également un rôle crucial dans la pensée de Husserl. Au début de la deuxième partie d'un de ses premiers travaux, Philosophie de l'arithmétique, Husserl attribue lui-même à Brentano l'inestimable distinction entre représentation propre ou authentique et représentation inauthentique ou symbolique. Husserl propose ensuite la définition suivante : 
Une représentation symbolique ou inauthentique est, comme le mot le dit déjà, une représentation par des signes. Si un contenu ne nous est pas donné directement, comme ce qu'il est, mais seulement indirectement par des signes qui le caractérisent univoquement, alors, au lieu d'une représentation authentique, nous avons de lui une représentation symbolique ${ }^{\mathrm{I}}$ (traduction modifiée).

La distinction entre « authenticité » et « inauthenticité » renvoie ultimement à la différence entre la pensée intuitive qui est caractérisée par l'immédiateté, par le fait de «voir clairement » ce qui est pensé et la pensée qui est symbolique ou médiatisée. Ce ne sont pas seulement les objets de la perception externe qui peuvent être donnés authentiquement ou inauthentiquement (par exemple, la différence entre voir l'image d'une maison et voir cette maison elle-même) car les concepts généraux ou abstraits peuvent également être donnés d'une façon ou de l'autre ${ }^{2}$. Le traitement que Husserl réserve à la différence entre « compter » et « calculer » (ou ce que Dallas Willard nomme « énumération sans réflexion $»^{3}$ ) aide à illustrer la distinction entre l'authenticité et l'inauthencité.

Compter, au sens propre, est un acte auquel appartient un certain contenu intuitif. Un adulte qui utilise la présence intuitive d'un objet sensible pour apprendre à un enfant à compter est un exemple possible pour illustrer l'acte propre de « compter ». Par contre, un tel besoin pour la présence des objets eux-mêmes est rapidement transcendé. En très bas âge, une personne normale a déjà développé une tendance à calculer numériquement, c'est-à-dire à inférer des conclusions et à conduire des opérations uniquement sur la base de symboles numériques, et ce, sans un besoin ou habituellement un intérêt pour le fondement intuitif des symboles ou pour la compréhension de la procédure inhérente au comptage authentique, procédure qui, selon Husserl, sous-tend tout calcul numérique.

Cette habileté à calculer est un très grand pouvoir de notre intelligence. En effet, sans le pouvoir de calculer, l'esprit humain

I. Edmund Husserl, Philosophie der Arithmetik, hrsg. von L. Eley, Husserliana XII, La Haye, Martinus Nijhoff, 1970, p. 193 (désormais Hua) ; tr. fr. J. English, Philosophie de l'arithmétique : recherches psychologiques et logiques, Paris, P.U.F., coll. Epiméthée, p. 236, les italiques sont dans la traduction mais pas dans les Hua.

2. Hua XII, P. I94

3. Dallas Wilard, Logic and the Objectivity of Knowledge, Athens, Ohio University Press, Ig84, P. I07. 
ne serait capable que d'un très léger progrès arithmétique. De plus, la pensée symbolique ou inauthentique tend vers une très grande « efficacité ». Elle permet d'obtenir d'excellents résultats à partir d'un minimum d'effort et elle est certes représentative du principe d' «économie de pensée » de Avenarius et Mach, auquel Husserl s'est beaucoup intéressé dans ses Recherches logiques ${ }^{4}$. L'habileté qu'a la pensée d'《 économiser », de ne pas avoir à retracer chaque pas menant à son origine intuitive, joue un rôle central dans le progrès rapide que la science est capable d'effectuer. Il y a par contre un aspect négatif à ce pouvoir. L'habileté pour le calcul est certes économique mais il implique également une certaine « cécité ».

Le danger est dû au fait que même si les représentations symboliques ne sont possibles que sur la base de la représentation propre, la nature fondée et dérivée des concepts « inauthentiques » a tendance à être « omise » ou « oubliée ». De plus, la façon d'utiliser les concepts inauthentiques peut aussi être oubliée. Cet oubli aide effectivement le progrès rapide des mathématiques et Husserl ne suggère aucunement qu'il ne faut pas calculer! Il déclare que toutes les cultures qui ont surmonté le barbarisme ont développé un système numérique symbolique ${ }^{5}$. Par contre cette tendance à l'oubli de la pensée inauthentique peut devenir « hors de contrôle ». En effet, ce que Husserl décrit plus de quarante ans après Philosophie de larithmétique dans son ouvrage certainement le plus lu, La crise des sciences européennes et la phénoménologie transcendantale, n'est rien d'autre qu'une crise engendrée par cette tendance à l'oubli.

La compréhension par Husserl de l'authenticité et de l'inauthenticité reste ambivalente. D'une part, la pensée inauthentique comporte de nombreux avantages : elle est nécessaire au progrès, elle apparaît presque intrinsèque à la science et elle caractérise les cultures évoluées. Par contre, lorsque la pensée inauthentique ou symbolique n'est plus liée aux intuitions et à la conscience authentique, elle perd le droit d'être nommée « science » et ne devient ainsi pour Husserl qu'une simple technique. Ceci n'empêche nullement la science de « fonctionner », en

4. Edmund Husserl, Recherches logiques, Paris, P.U.F., coll. Epiméthèe, I969, $\$ \S 52-56$.

5. Hua XII, p. 272-273.

6. Voir Rudolf Bernet, "Differenz und Anwesenheit ; Derridas und Husserl Phänomenologie der Sprache, der Zeit, der Geschichte, der wissenschaftlichen 
fait, cela lui permet même de devenir toujours plus active. Mais Husserl nomme « activité en passivité ${ }^{6}$ ce fonctionnement inauthentique. Avec ce fonctionnement, lacte se fait sans véritable intuition de ce que l'on fait, on agit sans être complètement conscient de la signification de nos actions, sans véritable compréhension de notre acte. Husserl était de plus en plus convaincu que malgré toutes les proclamations de maîtrise que la science avait faites et toute l'efficacité omniprésente des techniques de manipulation avancées, la science devenait en fait appauvrie au niveau de sa conscience réflexive et asservie à l'« activité en passivité ».

De plus, le danger encouru lorsque la pensée inauthentique devient « hors de contrôle » et qu'elle se caractérise comme «activité en passivité » dépasse largement le domaine de la science. Dans son essai de 1936, L'origine de la géométrie, Husserl développe une conception du langage très similaire à ce qui vient d'être dit sur les nombres ${ }^{7}$. Pour Husserl, la philosophie est ellemême inauthentique lorsqu'elle pratique l'activité en passivité, que ce soit par des prétentions non questionnées issues du passé ou par le maintien plus subtil au sein de la tradition de préjugés non remarqués et de points de vue bornés et incomplets. Husserl indique également la religion comme exemple potentiel d'une inauthenticité qui devient « hors de contrôle ». La passivité et le traditionalisme qui caractérisent la religion naïve et naturaliste peut apparaître au sein de la religion de l'âge moderne, et Husserl ajoute sans détour que celle-ci est « la religion des églises conventionnelles ${ }^{8}$.

Rationalität », dans Studien zur neueren französischen Phänomenologie. Phänomenologische Forschungen, Band 18, Fribourg, Karl Alber Verlag, 1986, p. 100 ff.

7. Cet essai fut publié suite à la mort de Husserl par Eugen Fink sous le titre « Die Frage nach dem Ursprung der Geometrie als intentional-historisches Problem » dans Revue intemationale de philosophie, I, 1939, p. 203-225. D'après son contenu, il a toujours été lu dans le contexte de la problématique de la « Krisis » et ce texte peut être retrouvé dans l'appendice III de La crise des sciences européennes et la phénoménologie transcendantale, Paris, Gallimard, 1976, p. 403-427 lensuite cité Hua VII. Ce texte parut initialement en français comme L'origine de la géométrie, traduction et introduction de Jacques Derrida, Paris, P.U.F., I962. Cela est bien connu, ce texte est luimême l'origine d'une grande partie de ce qui est intéressant dans le travail de Derrida. Par contre, Derrida ne conçoit pas l'aspect « hors de contrỏle » du langage comme un danger, ou plutôt, il le considère comme un danger essentiel au langage et contitutif de celui-ci. Il met ainsi l'accent sur une ambiguité propre au traitement husserlien de l'authenticité et l'inauthenticité.

8. Edmund Husserl, Aufsätze und Vorträge (1922-1937), hrsg. von T.Nenon und H.R. Sepp, 
Si l'inauthenticité est caractérisée comme « activité en passivité » alors l'authenticité peut être décrite pour Husserl comme une « activité en activité ». L'inauthenticité peut toujours être dépassée par la volonté courageuse et active de ne pas vivre dans la passivité, et ceci, par un effort constant visant à justifier nos « prises de positions » [Stellungnahmen] tant théoriques que pratiques sur la base d'évidences, à travers ce que Husserl nomme à un certain moment l'« héroïsme de la raison $»^{9}$. Selon Husserl, la vie humaine authentique consiste à se retirer hors de la vie simplement guidée par un courant de tendances irrationnelles, par toutes sortes de validités pré-données et de former une vie basée sur le choix critique ${ }^{\text {IO }}$.

L'acquisition de cet habitus ${ }^{\mathrm{II}}$ de la critique, de la compréhension et de la justification des positions de chacun ne peut être fondée que sur un effort volontaire, un choix visant à donner une nouvelle direction à notre vie ${ }^{\mathrm{I} 2}$. Toutes les manières différentes de surpasser l'inauthenticité sont basées sur la décision de se libérer constamment et consciemment de l'activité « passive » et irréfléchie caractéristique de la vie inauthentique. On ne traitera jamais assez de la notion de « résolution de la volonté » dans le contexte de l'authenticité. Non seulement l'authenticité est une décision de vivre d'une certaine manière mais c'est une décision qui doit constamment être réitérée dans la vie de chacun. La notion d'habitus exprime très bien cette constance, car elle ne réfère pas à une simple décision ou à un acte noble mais plutôt à un mode de vie maintenu continuellement, toujours et partout, peu importe les circonstances.

La conception husserlienne de l'authenticité repose clairement sur une forme de volontarisme. L'idée suivant laquelle l'authenticité humaine implique une volonté de comprendre s'appliquant autant au domaine théorique qu'au domaine pratique sous-tend une conception liant la vie humaine authentique à un

Husserliana XXVII, Dordrecht, Kluwer Academic, 1989, P. I23.

9. Hua VI, p. 348 .

10. "Leben ist in sich Streben und ist als menchlisches ein Aufsteigen von Passivität in Aktivität, von einem Sich-gehen-und -tragen-lassen, von einem Leben nach Neigung, zu einem überlegten Wählen, zu einem kritischen, höher bewertetes bevorzugenden Leben ». Archives Husserl $F$ I 24 / 7 ob (1933). Je remercie Ullrich Melle pour les échanges que nous avons eus autour de ce manuscrit.

II. Hua XXVII, p. 64.

12. Hua VI, p. $147-148$. 
perpétuel combat contre des impulsions et des croyances irrationnelles, contre ce qui n'a pas été choisi et voulu de façon rationnelle par le sujet. Dans un manuscrit de son cours de igzo consacré à l'éthique, Husserl suggère que le désir pour une vie totalement rationnelle opère une division de l'ego en un ego supérieur de la raison et un ego faible et coupable à la merci de ses impulsions ou de validités pré-données. La vie humaine authentique est un Kampf à la fois contre les impulsions individuelles et les tendances sociales allant à l'encontre d'une auto-détermination rationnelle du sujet par le sujet.

La définition classique de l'homme comme animale rationale peut prendre une signification particulière pour Husserl. Ce terme peut être considéré comme une découverte de la différence entre le vrai soi et le faux soi ou comme un symptôme du combat se menant au sein du soi. L'animal est le soi inférieur, le soi de la passivité, de l'inconscience, du fonctionnement à vide et insignifiant, bref, un soi privé de compréhension. Mais il y a un soi supérieur qui est, pour Husserl, le soi véritable. Le soi supérieur est celui de la raison, un soi rationnel et habité par l'habitus de la critique et par la volonté de connaître et de comprendre. Si la vie humaine peut être caractérisée comme un combat entre deux égoités, le but de la vie humaine authentique devrait consister en un triomphe de l'ego supérieur.

L'aspect radical de l'affirmation de Husserl déterminant l'authenticité comme souveraineté du rationnel, ou comme prédominance du soi actif sur le soi passif, est manifeste dans sa conception de la « sainteté ». Pour Husserl, une personne qui fait le bien sans savoir pourquoi elle le fait mène une vie ancrée dans la passivité et l'inauthenticité. Un tel mode de vie représente une situation où le soi supérieur demeure asservi au soi inférieur. Conséquemment, le « saint » ou une personne qui se limite à faire le bien est comparée par Husserl à ce qu'il nomme « un gentil petit animal $\gg$ [eingutes Tierchen $]^{\text {13 }}$. Même si cette personne effectue des actes voués au bien, elle demeure un « animal » passif. Un «saint » humainement authentique est une personne qui fait le bien et qui sait pourquoi elle le fait. Le saint humainement authentique est une personne qui fait le bien conformément à une réflexion et une compréhension du bien lui-même, non

13. Manusctit Archives Husserl B I 2I/7a. 
seulement avec le désir de faire le bien mais avec celui de le « connaître ». Pour Husserl, le véritable soi sait ce qu'il fait et il sait pourquoi. Il est donc entièrement responsable de ses actes et il peut répondre de ceux-ci. Pour cette raison, la meilleure description de l'authenticité est la responsabilité de soi et l'ultime caractérisation de la philosophie husserlienne est celle de la responsabilité absolue de soi.

\section{Heidegger et l'authenticité}

Une des difficultés que l'on rencontre en examinant le traitement par Heidegger de l'authenticité et de l'inauthenticité est redevable du fait que la rhétorique de son discours ne correspond pas toujours à la nature dite « formelle » ou purement descriptive des affirmations. Dès le début de Être et Temps, Heidegger pose clairement que l'authenticité et l'inauthenticité ne correspondent pas à des états moraux. L'inauthenticité du Dasein « ne signifie pourtant pas une sorte de "moindre" être ou un niveau d'être "dégradé" »I4. L'authenticité et l'inauthenticité sont deux modes par lesquels le Dasein est dans le monde, et ces deux modes ne sont possibles que sur la base de la temporalité essentielle du Dasein $^{\text {I5 }}$. Par contre le ton utilisé pour décrire ces deux états ne rend pas toujours compte de cette supposée « égalité » morale et ontologique. Gadamer, qui était présent lors du premier exposé de Heidegger portant sur la différence entre l'authenticité et l'inauthenticité, trouvait très difficile d'écouter les invectives de Heidegger contre l'inauthenticité pour ensuite accepter, suite à l'insistance de Heidegger, que rien de négatif n'était signifié par ces invectives ${ }^{16}$ !

La relation structurelle entre l'authenticité et l'inauthenticité pose également quelques problèmes. Lors d'un certain passage de Être et Temps, Heidegger déclare que «l'inauthenticité est fondée sur la possibilité de l'authenticité $\gg^{17}$. Une telle affirmation correspond à l'herméneutique par laquelle la temporalité du Dasein fut originellement découverte. C'est sur la base du souci authentique que la découverte de la signification ontologique du Dasein

14. Martin Heidegger, Être et Temps, Paris. Gallimard, 1986 Icité ensuite ÊT], p. 74.

15. $\hat{E} T$, p. 363

16. Hans-Georg Gadamer, Philosophische Lehrjahre, Francfort, Vittorio Klosterman, 1977, p. $214 \mathrm{ff}$.

17. $\hat{E} T$, p. 313 . 
comme temporalité est advenue. Par contre, une telle affirmation semble également placer la problématique authenticité / inauthenticité dans un cadre que la pensée de Heidegger rend problématique. Cette affirmation semble suggerer une sorte d'authenticité primordiale par laquelle advient la possibilité de sa perte, une possession de soi originelle à partir de laquelle surgit la perte de soi et l'inauthentique séparation de soi.

Par contre, Heidegger mentionne également que l'authenticité n'est pas quelque chose de séparé de l'inauthenticité mais plutôt une 《modification $\gg^{18}$. Suite à la juxtaposition de ces deux affirmations, nous nous devons de conclure que l'authenticité est autant la condition de possibilité que la modification de l'inauthenticité. Certains commentateurs philosophes y voient une contradiction et Michel Zimmerman affirme même qu'il s'agit là d'une pure impossibilité ${ }^{\text {I9 }}$. Ce point de vue est beaucoup trop radical. La manière habituelle par laquelle le Dasein est dans le monde rend celui-ci complètement inconscient de son « véritable » soi et de la nature propre de son être-au-monde. Lorsque le Dasein est tiré hors de l'indifférence, lorsqu'il développe l'impression qu'il n'appartient pas entièrement au « on », il fait alors l'expérience de $\mathrm{l}$ ' «authenticité » comme une « modification » de son existence inauthentique précédente. Mais il ne faut pas oublier qu'une telle reconnaissance n'est possible que sur la base de l'authenticité; seul un Dasein authentique reconnaît sa propre inauthenticité. Le Dasein peut faire l'expérience de la modification de l'inauthenticité lordo cognoscendil, ce qui n'empêche pas que l'authenticité demeure la base ontologique d'une telle reconnaissance lordo essendi $]^{20}$. Ia notion « monde de la vie »développée par Husserl peut nous aider à saisir ce mouvement à deux niveaux.

Selon Husserl, le monde de la vie est le monde du quotidien au sein duquel nous menons notre vie, une vie pleine de validités non questionnées et de présuppositions ontologiques naïves. Les sciences naturelles et humaines prétendent nous informer au sujet de ce monde. Par contre, non seulement ces sciences sont

\footnotetext{
18. ÊT, p. 173 .

I9. Michael Zimmerman, Eclipse of the Self : the Development of Heidegger's Concept of Authenticity, Athens, Ohio University Press, 1981. p. 45 .

20. Je remercie Rudolf Bernet pour avoir clarifier la situation en suggérant les termes ordo cognoscendi et ordo essendi.
} 
infectées par la tendance à reconnaître toutes sortes de «validités » non questionnées, de même que par le maintien de présuppositions ontologiques non fondées, mais elles dissimulent même leurs propres fondements concrets dans le monde de la vie. Au lieu de révéler la nature du monde de la vie, elles sont actuellement en train de l'enfermer dans un «vêtement d'idées ». Cependant, comme nous l'avons mentionné précédemment, Husserl n'affirme aucunement que la vie simple et non scientifique dans le monde soit la forme de vie la plus « authentique». Une naïveté est inhérente à la vie ordinaire dans le monde, une certaine perte de notre conscience propre. Même si ce n'est pas l'obscurité construite par les sciences et leurs idéalisations, nous retrouvons également une obscurité propre à la vie ordinaire dans le monde. En langage husserlien, les diverses activités constituantes ne sont pas évidentes pour le sujet engagé dans la vie quotidienne, car il ne réalise pas qu'il est l'origine constitutive du monde. La vie réellement authentique consiste à dévoiler la nature transcendantale de la vie dans le monde. Ce faisant, le sujet devient habilité à connaître ce qu'il fait pour ainsi s'offrir une position lui permettant d'être responsable de lui-même et du monde de la vie.

Selon Husserl, l'existence quotidienne dans le « monde de la vie $\gg$ et celle dans le monde de la science sont toutes deux aveugles à la vraie nature transcendantale de l'existence humaine. Ia différence entre les deux est attribuable au fait que la première peut être qualifiée d'aveuglement « non dogmatique » tandis que l'aveuglement de la science est «dogmatique ». Les présuppositions ontologiques de la vie quotidienne n'ayant pas été remarquées ou examinées apparaissent en effet « naturelles ». Le voilement de ce que les propositions ontologiques de la vie quotidienne cachent est prévisible. Dans l'autre cas, nous pouvons dire que les sciences renferment un contenu d'inauthenticité dogmatique à l'aide duquel la naïveté du monde quotidien est transformée en témoignage explicite de «fidélité ». Les sciences ne sont pas exclusivement naïves, mais elles élaborent un schème d'interprétation fondé sur cette naïveté. Les énormes constructions théoriques des sciences humaines et naturelles sont en fait des obstructions qui sont en quelque sorte « pires » que ce qui est élaboré dans la vie quotidienne car les points de vue obstructifs de la science sont reçus comme des « révélations ». 
Heidegger a sans aucun doute quelque chose de similaire en tête lorsqu'il distingue la «quotidienneté » de l'inauthenticitéz ${ }^{2 I}$. L'indifférence quotidienne du Dasein est inauthentique, mais cette inauthenticité ne recèle aucun niveau de conscience de soi. Il y a par contre une vie inauthentique qui prétend faire appel à une conscience de soi très développée. Par contre, cette conscience de soi n'est pas réelle. Il s'agit d'une conscience de soi propre au niveau « ontique », la conscience de soi de l'être « au sein du temps ». Là encore, la différence peut être exprimée dans les termes d'une corrélation comprenant, d'une part, l'inauthenticité dogmatique et, d'autre part, une inauthenticité non dogmatique.

Tant pour Husserl que pour Heidegger, l'authenticité est relative à une thématisation adéquate de ce qui reste non thématisé dans la vie ordinaire ou dans la vie scientifique, rendant ainsi explicite ce qui n'était qu'implicite. Ce qui n'est d'aucune façon remarqué dans l'existence quotidienne et indifférente, c'est, pour Heidegger, la signification ontologique du Dasein comme temporalité. Cette temporalité ne peut se « temporaliser » elle-mème que de deux façons ; authentiquement (devancement-répétition-instant) ou inauthentiquement (attendre-oublier-présentifier) ${ }^{22}$. Dans l'« indifférence » quotidienne, il y a un manque complet de cette signification ontologique du Dasein.

L'authenticité est une modification de la quotidienneté indifférente car elle s'effectue par la résolution de faire face à ce que l'indifférence quotidienne cache : le Dasein comme temporalité finie. Mais la nature obstructive de cette indifférence ne peut apparaître que du point de vue de l'authenticité, elle ne peut devenir consciente d'elle-même qu'à travers l'authenticité. En ce sens, l'inauthenticité advient hors de l'authenticité. Maintenir que la quotidienneté est la structure de toute existence ne revient pas à suggérer qu'il y a un troisième type de position qui sous-tend l'authenticité et l'inauthenticite ${ }^{23}$. Il s'agit plutôt d'accentuer le

21. Martin Heidegger, Iogik : Die Frage nach der Wahrheit, hrsg. von W. Biemel, Gesamtausgabe 21, Francfort. Vittorio Klosterman, 1976, p. 229-230. En d'autres occasions, il conçoit une égalité entre la quotidienneté et l'inauthenticité, et il oppose ces deux termes à l'authenticité, Heidegger, Les problèmes fondamentaux de la phénoménologie, Paris, Gallimard, 1985, p. 198.

22. $\hat{E} T$, p. 363

23. La conclusion de Michael Zimmerman, (op. cit. p.46), entraîne qu'il y ait trois modes d'existence plutôt distincts les uns des autres. Ceci semble également être l'argument de Michel Haar losqu'il prétend qu'il y a un « oubli originaire » 
fait que le Dasein ne quitte jamais son $D a$ et que sa quotidienneté insouciante appartient essentiellement au Da du Dasein. Dire que l'authenticité est à la fois modification et condition de possibilité de l'inauthenticité indique la corrélation entre les deux et implique une constante interaction de ces deux états. Cela suggère également que l'existence ne peut jamais être complètement ou purement authentique.

L'inauthenticité qui concerne le plus Heidegger est celle menant à l'oubli de la question du sens de l'être. Comme le Dasein est temporel, cet oubli est rattaché à un mode inauthentique de temporalisation et en particulier à une attitude inauthentique envers le passé. Mais étant donné qu'aucune des exstases temporelles ne sont isolées, cet oubli est relié à une attente inauthentique du futur et à un « présentifier ». Le dépassement de cette inauthenticité correspond à une approche authentique de la tempolarisation et, en particulier, à la « répétition 》 (Wiederholung). Cette répétition, c'est-à-dire la répétition de la question du sens de l'être, peut également être considérée comme une répétition du questionnant, une répétition du soi hors du « on » et ce, par la réalisation du Dasein de sa propre potentialité à soulever la question de l'être. Là encore, cette répétition ne peut jamais être complètement dégagée des autres extases temporelles car elle recẻle elle-même un sens référant essentiellement au futur. Par contre, ce qui est également évident pour la pensée de Heidegger, c'est que la répétition ne peut jamais être complètement séparée de l'oubli.

Malgré toute l'emphase mise sur le lien étroit entre la répétition et l'oubli, la tendance à penser qu'une doit précéder l'autre ne disparaît pas. De façon claire et directe, nous pourrions dire que pour que quelque chose soit répété ou remémoré, cela doit d'abord avoir déjà été oublié. Cependant, pour que quelque chose soit oublié, il doit, en quelque sorte, avoir déjà été « présent ». Il peut y avoir une certaine circularité entre la répétition et l'oubli mais il n'apparaît pas ultimement qu'il s'agisse d'un

qui précède l'inauthenticité. Au contraire, je prétends que l'« oubli originaire » renvoie à l'inauthenticité qui « soutient » le Dasein dans sa quotidienneté. C'est sur cette inauthenticité fondamentale qu'est fondée la notion dérivée et inauthentique du ressouvenir (Erinnerung) et de l'« oubli », au sens commun de ces termes, et c'est précisément cette inauthenticité que s'approprie l'authenticité. Cf. Michel Haar, Heidegger et l'essence de lhomme, Grenoble. Jérôme Millon, 1990. p. 79. 
cercle totalement clos; ce cercle semble nous ramener à un point originel, une origine où le questionnant était en possession de lui-même et ainsi de sa possibilité la plus propre, possibilité lui permettant d'aborder la question du sens de l'être. La conclusion serait donc qu'il y a un certain point précédant temporellement l'occurrence de l'oubli.

Devons-nous considérer la perte du Dasein dans le « on » comme la perte d'une possession de soi primordiale? Il est certain que Heidegger répondrait négativement à cette question car cet oubli est une possibilité existentielle du Dasein, une possibilité « équiprimordiale » (gleichursprünglich) par rapport à la possibilité de la répétition menant hors du « on ». Comme Heidegger le dit lui-même à l'occasion d'un exposé présenté quelques années après la parution de Etre et Temps :

Nous commençons notre existence avec un tel oubli de la compréhension de l'être, et plus nous nous ouvrons aux ètants, l'oubli de ce que nous comprenons dans toute ouverture comme l'être de l'étant devient étendu ${ }^{24}$.

Le Dasein est l'étant ayant une ouverture particulière à l'être, mais cette ouverture est liée dès son occurrence à un certain «voilement ». Ainsi, le Dasein perdu parmi les étants auprès desquels il est jeté n'arrive pas à se saisir lui-même comme l'unique étant possèdant la possibilité de questionner l'être.

Le même genre de questions soulevées à propos de l'authenticité du Dasein particulier doivent être soulevées à propos de la tradition philosophique : si elle consiste en une interaction entre l'oubli et la répétition, n'y aurait-il pas lieu de croire que ce cycle ait débuté par une sorte de compréhension pure de l'être? Malgré les déclarations de Heidegger sur la nécessité de l'oubli, il apparaît que cet oubli consiste en une « dégénération », quoique cette dégénération puisse être considérée comme nécessaire. La façon dont Heidegger parle de l'« origine », de la « source », de ß« occurrence fondamentale » nous renvoie directement à la nécessité de « récupérer l'expérience originelle de l'être $»^{25}$ et elle suggère une sorte de répétition d'un moment précédant l'oubli.

24. Martin Heidegger, Vom Wesen der menchlischen Freiheit, hrsg. von H. Tietjen, Gesamtausgabe 3I, Francfort, Vittorio Klosterman, 1976. p. 42.

25. Martin Heidegger, "Zur Seinsfrage », dans Wegmarken, hrsg. von F.W. von Hermann, Gesamtausgabe 9. Francfort, Vittorio Klostermann, 1976, p. 417 ; tr. fr.: «Contribution à la question de l'être», dans Questions I et II, Paris, Gallimard, Ig68. 
Cette impression d'arriver à un point précédant l'occurrence de l'oubli n'est pas seulement présente dans le contexte de la question de l'être mais également par rapport à d'autres types de répétitions se présentant dans la pensée de Heidegger. Par exemple, nous verrons à la quatrième section de cet article que lorsque le jeune Heidegger développe une répétition des débuts du christianisme, il se retourne vers les Épîtres aux Thessaloniciens, car dit-il, celles-ci font partie des documents les « plus originels » du christianisme, précédant même les Évangiles. Par rapport à l'oubli se manifestant à travers un usage irréfléchi du langage, Heidegger suggère (de façon similaire à ce que nous avons vu chez Husserl) « un retour au contenu originel du langage qui nous est propre et que nous avons conçu, langage en constante dégénération $»^{26}$. Il est certainement à propos ici de se concentrer sur la notion de « contenus originels ». La notion de dégénération constante est par contre tout aussi importante. Il s'agit d'une dégénération présente dès le début et à laquelle on ne pourra jamais se soustraire.

Ainsi, même si la pensée heideggerienne réfère fréquemment à une certaine « origine », à un « commencement ${ }^{27}$, le commencement consiste toujours lui-même en une interaction entre l'oubli et la répétition. Ceci explique la position constante de Heidegger voulant que si sa pensée doit être interprétée comme la description d'une Verfallsgeschichte, cela ne doit pas être entendu négativement ${ }^{28}$. En effet, il est propre à la nature de l'histoire, et certainement à l'histoire de l'être de « dégénérer », mais cette dégénération peut bien être considérée comme une opportunité, comme un don. S'est-il produit une erreur dans la tradition philosophique? Oui mais là où s'est produite l'erreur résidaient les plus grandes possibilités pour soulever la question de l'être. Dans le langage du « demier » Heidegger, la tradition est un « avènement », une « appropriation » [Ereignis] qui inclut nécessairement la perte, une histoire de la propriation ler-eignenl qui est également une histoire de la désappropriation lent-eignen $\left.\right|^{29}$. Cet

26. Günther Neske and Emil Kettering (dir.). Antwort : Martin Heidegger im Gespräch Pfullingen, Günther Neske Verlag, I988, p. 28.

27. « Nun aber ist das grösste Ereignis immer der Anfang ». Martin Heidegger, Beiträge zur Philosophie (Vom Ereignis), hrsg. von F.W. von Herrmann, Gesamtausgabe 65. Francfort, V. Klostermann, 1989. P. 57.

28. Neske, Kettering (dir.), op. cit., p. 23.

29. Cette interaction entre la perte et le gain est très bien amenée dans le contexte de 
oubli peut être une occasion de félicité, un «felix culpa », car elle peut éventuellement mener à la possibilité de la répétition, à l'authenticité.

Si l'inauthenticité est présente depuis le début comme un moment essentiel de la « vérité », elle pourrait être « inévitable». Parallèlement, il semblerait que l'authenticité soit au-delà du contrôle propre au Dasein. Cela est-il vrai pour Heidegger ? Les moments de l'authenticité et de l'inauthenticité sont-ils tout deux inévitables et au-delà de notre contrôle ou Heidegger souhaite-t-il secrètement un dépassement volontaire de l'inauthenticité par le Dasein authentique? Dit autrement, l'inauthenticité est certainement redevable à l'échéance du Dasein et à sa tendance au voilement. Le Dasein est enclin au contentement, à se perdre luimême parmi les choses du monde, à s'oublier lui-même comme l'unique étant ayant une ouverture particulière à l'être. Le Dasein est en proie à l'échéance, il n'échappera jamais complètement à cette tendance. Par contre, à certains moments, le Dasein a une impression du caractère unique de son propre être, de ce qu'il est en tant qu'ouverture finie à l'être. C'est l'auto-compréhension qui caractérise l'authenticité. Comment advient cette « auto-compréhension »? Est-elle vraiment « acquise » par le Dasein ou est-elle en quelque sorte imposée au Dasein de l'« extérieur »? L'authenticité est-elle un choix ou le Dasein est-il en quelque sorte choisi? L'authenticité est-elle un accomplissement volontaire ou un don précieux?

\section{Le volontarisme heideggerien et l'interaction passil / actif au sein de l'authenticité}

Étant donné l'ambivalence présente à tous les niveaux de la pensée heideggerienne, il n'est pas surprenant que l'« accomplissement » de l'authenticité comporte deux aspects. L'authenticité advient à la fois par l'accomplissement et par la résignation, sous la forme d'un choix qui inclut de se laisser choisir, un accomplissement qui n'est jamais complètement redevable au Dasein. Décrite de façon générale et conformément aux termes établis précédemment lors de l'examen de la pensée husserlienne, l'authenticité est pour Heidegger une activité accompagnée d'une

la « mimésis », voir Samuel Ijsseling. Mimesis : Over schijn en zijn, Baarn, Ambo, 1990, p. $47-60$. 
passivité fondamentale. Heidegger mentionne lors d'une discussion portant sur l'état authentique de la résolution qu'il est hésitant à utiliser le terme « agir » en ce qui a trait au Dasein résolu. Car, pour utiliser ce terme correctement, « il faudrait arriver à le recomprendre assez globalement pour que l'activité englobe jusqu'à la passivité de la résistance ». Comment devonsnous alors comprendre cette activité incluant la passivité et que peut-elle nous révéler sur l' « habilité » du Dasein à « devenir » authentique $^{30}$ ?

L'indication la plus claire nous menant à croire que l'authenticité est plutôt ressentie qu'inhérenteà une situation où elle aurait été accomplie vient d'une déclaration de Heidegger posant que la possibilité pour un changement de la temporalité sur lequel est fondée l'authenticité s'annonce elle-même sous le type d'une Befindlichkeit appelée « angoisse ». En effet, l'angoisse joue un rôle si important pour Heidegger qu'il la nomme «GrundBefindlichkeit » ${ }^{3 \mathrm{I}}$. Lorsqu'il est $\mathrm{d}^{\prime}$ 《 humeur » anxieuse, le Dasein sent le monde se dérober sous ses pieds et fait ainsi l'expérience de l'aspect incontournable et étrange d'un monde qu'il considère généralement comme un fait acquis. Durant les moments d'angoisse, ou pendant qu'il réalise qu'il « n'est pas chez lui dans le monde », le Dasein subit une perte de la sécurité et du confort habituellement obtenue par son appartenance au « on ».

Il est par contre important de reconnaître que Heidegger s'efforce d'expliquer que le Dasein n'est pas angoissé au sujet de quelque chose mais plutôt de lui-même et du type d'être qu'il est lui-même, un être de temporalité finie. Comme le dirait Pascal, nous sommes effrayés de qui nous sommes, de la mortalité qui nous est essentielle ${ }^{32}$. Lorsqu'il est en proie à l'angoisse, le Dasein ne « craint » rien en particulier dans le monde mais il « fuit » plutôt devant l'« être-au-monde comme tel » et entre autres, devant le Dasein lui-même ${ }^{33}$. Mais cette fuite est vaine car le refuge normal du Dasein, la certitude et le confort de la vie publique au sein du « on » est précisément ce qui est éliminé par l'angoisse. Cette dernière remarque met en relief la fonction «positive » que nous pourrions attribuer à l'angoisse car elle rend inefficaces les

30. $\hat{E} T$, p. 359 .

3I. ÊT, p. 230.

32. Francis Kaplan (èd.), Les Pensées de Pascal, Paris, Editions du Cerf, I982, p. 192-202.

33. ÊT. p. 234-235. 
moyens d'auto-compréhension que le Dasein retire de sa présence au sein du « on », situation qui amène le Dasein à se confronter lui-même. En effet, l'angoisse comporte un aspect « libérateur » pour le Dasein $^{34}$ car elle lui permet de devenir libre pour son propre type d'être, faisant ainsi place à une auto-comprẻhension adéquate, place à l'authenticité.

Être angoissé est en effet une expérience « passive» paradigmatique. L'angoisse n'est pas voulue, attendue ou contrôlable. Par contre, il est crucial de garder à l'esprit que d'être dépassé ou subjugué par l'angoisse n'est pas authentique. Cette Befindlichkeit de base qui ouvre un espace à l'authenticité doit néanmoins être accompagnée d'éléments « actifs ». L'authenticité est alors relative à la conscience active de ce qui se produit dans l'angoisse, à la classification et la thématisation de cette expérience passive.

Une analyse de l' « appel » de la conscience développée par Heidegger aide à clarifier cette interaction actif / passif. La conscience appelle le Dasein à maîtriser l'angoisse qui fait surface lorsqu'il ressent l'inévitabilité de sa propre perte, pour faire face à ce qu'il fuit habituellement, entre autres lui-même et sa propre temporalité finie. La conscience appelle le Dasein à accepter résolument sa propre finitude, sa propre mortalité. Cette résolution, authentique acceptation du Dasein lui-même, est décrite par Heidegger comme « se laisser appeler ${ }^{35}$, ou l'écoute face à l'appel. Par contre, lorsque nous considérons la dynamique inhérente à un « appel », des éléments propres à l'activité apparaissent. Premièrement, pour qu'un appel soit entendu, il doit préalablement être émis. Deuxièmement, le récepteur doit être dans une situation propice pour recevoir l'appel. Finalement, entendre un appel ne signifie pas « répondre ». Au premier point, nous devons ajouter que l'émetteur ou l'origine de cet appel est le Dasein lui-même. L'émission de cet appel est en-soi un bon exemple du lien entre l'activité et la passivité car comme le dit Heidegger, « l'appel vient de moi tout en me tombant dessus ${ }^{36}$. L'émission de l'appel ne doit en aucune façon être interprétée comme une décision d'appeler. Par contre, il ne vient pas de l'extérieur au sens d'une présence objective. L'appel est une forme d'auto-affection, un appel du soi au soi, 
et d'un autre point de vue, la réception pour le Dasein de quelque chose qu'il se donne à lui-même.

Pour qu'un appel soit entendu, nous devons être dans une position propice, un rôle encore plus actif semble être ici attribué au Dasein. Il est vrai que le Dasein est « convoqué », mais il doit cependant « se laisser » convoquer. Ce n'est que le Dasein ouvert à l'authenticité qui « se donne la situation chaque fois factice et qui $s^{\prime}$ immisce en elle ${ }^{37}$. Dit autrement, le Dasein est en quelque sorte responsable de sa perte parmi les choses du monde, ou encore mieux, il est responsable d'avoir laissé cette perte devenir complète, dominante, ne laissant ainsi aucune ouverture à la réalisation du caractère unique de son propre être. Certes, le Dasein est également responsable d'avoir provoqué la rencontre avec le soi, non pas parce qu'il peut faire cela par la force de la volonté, mais parce qu'il peut se préparer à faire face à l'angoisse.

Finalement, en ce qui concerne la « réponse » à l'appel, la description que donne Heidegger de la résolution du Dasein comme acceptation de sa propre mortalité est en grande partie constituée de langage hautement volontariste, ce qui pourrait nous faire croire que la résolution est fondée sur une décision et non sur un appel. Le Dasein doit se saisir lui-même et saisir ses propres possibilités. Ceci devient plus évident lorsque nous considérons le Dasein par rapport à son historicité comme concrétisation de sa propre temporalité. Dans ce cas, le Dasein authentique doit choisir ses héros et résolument suivre leurs traces. C'est précisément le Dasein qui est « aveugle face aux divers choix ${ }^{38}$, qui est perdu dans le « on » à un point tel qu'il n'a plus de choix réels. L'angoisse et l'écoute de sa propre conscience libèrent le Dasein, ce qui lui permet de choisir mais il doit par contre agir conformément à cette possibilité d'authenticité apparaissant devant lui.

\section{N. La foi et la création artistique comme modèles de vie authentique}

Une façon de saisir cette interaction entre l'activité et la passivité est d'examiner les modèles d'authenticité présents à l'arrière-plan de la pensée heideggerienne. La répétition, développée par Heidegger, de la compréhension de « fidélité » et de 
«culpabilité » propre au début du christianisme, est sùrement une source pouvant éclairer ce qu'il entendait par vie authentique. Moins d'attention fut portée à cet aspect de la pensée de Heidegger qu'à sa reconstitution du « premier commencement » de la philosophie grecque et de son importante détermination pour l'histoire de l'ontologie. Néanmoins, cette reconstitution des débuts du christianisme fut très importante pour Heidegger tant à ses débuts à Fribourg qu'à Marbourg ${ }^{39}$. Un des documents les plus importants pour cette répétition est le cours du semestre d'hiver I920-rg2i donné à Fribourg intitulé : « Introduction à la phénoménologie de la religion $\gg$.

L'introduction de ce cours porte sur ce que Heidegger nomme « expérience de la vie factice » [faktische Lebenserfahrungl, une expression qui présage la notion d'existence factice ou plus simplement, le Dasein lui-même dans Être et Temps. La raison d'être de cette description de l'expérience de vie factice apparaît dans la deuxième partie, la plus élaborée de ce cours dans laquelle Heidegger se penche sur l'Épitre de Paul aux Galates et sur les deux Epitres aux Thessaloniciens. Selon Heidegger, les débuts du christianisme avaient une forte impression, quoique opaque, de l'expérience de vie factice et de sa temporalité propre.

Pour le christianisme du début, l'expérience se présentait sous deux formes fondamentales : la culpabilité et la fidélité. La distinction à la Bultman qu'introduit Heidegger entre l'authenticité et l'inauthenticité peut être considérée comme une « démythologisation $\gg$ [Entmythologisierungl de la distinction entre fidélité et culpabilité ${ }^{41}$. La culpabilité est le produit d'une surenchère du soi et de notre volonté propre, elle est basée sur le fait que «je »contrôle les choses du monde qui sont là pour que «je »

39. Il est important de noter que l'intérêt qu'il portait à ces textes ne devrait nullement être interprété comme de la Chrétienneté « confessionnelle ». Cela est très bien documenté par Hugo Ott. Martin Heidegger : Unterwegs zu seiner Biographie. Francfort, Campus, 1988 .

40. Ce cours n'est pas inclus dans la Gesamtausgabe, mais nous avons les sources nécessaires pour en tirer une bonne compréhension. Par exemple, les ArchivesHusserl à Leuven ont en leur possession un Nachschrift de l'étudiant Fritz Neumann. D'autres informations sont fournies par Otto Pöggeler, Der Denkweg Martin Heideggers, Pfullingen, Günther Neske Verlag, 1963, 1983. 1990, p. 36-45, et Thomas Sheehan, « Heidegger's Introduction to the Phenomenology of Religion » 1920-2I, The Personalist, 60, 1979. p. 312-324. La discussion qui suit est basée sur ces informations.

41. Zimmermann, op. cit., p. 58 sq. 
puisse en faire ce qui me plaît. Cependant, la culpabilité est également comprise comme «être perdu » parmi les choses du monde, même si cela se manifeste comme une volonté de possession des choses du monde, car le sujet se retrouvant dans cet état est, d'une certaine façon, lui-même possédé par ces choses. La culpabilité est facilement comparable à la notion d'« activité en passivité », une activité volontaire et centrée sur soi qui n'est possible que parce que le sujet ne choisit jamais réellement. Par contre, la fidélité est basée sur la prise de conscience que nous ne sommes pas le centre de l'univers, ni le seul et unique maître de notre propre vie. Elle consiste en une reconnaissance de soi pouvant être comparée à une perte du soi qui domine, qui contrôle et qui se conçoit comme maitre et centre du monde pour ainsi faire place à une réalisation de soi comme récipiendaire d'un don. Dans un langage théologique plus traditionnel, c'est la prise de conscience menant l'homme à ne plus se considérer comme créateur mais plutôt à s'accepter comme étant lui-même créé. La fidélité est donc une forme de découverte de soi.

A chacun de ces états appartient une temporalité particulière. Celui qui vise la maîtrise ne peut par contre jamais changer ou maîtriser le passé qui devient dans ce cas un fardeau, et la cause de la culpabilité ${ }^{42}$. Ou bien la maîtrise du présent ne s'effectue pas aussi bien que l'on aurait espéré, et dans ce cas, le passé devient un refuge, une galerie pleine de vestiges témoignant de triomphes et de dominations antérieures. Parallèlement, l'individu plein de bonne volonté peut tenter de contrôler le futur, de le planifier complètement et de le considérer comme une opportunité de manipuler toujours plus. Le coupable « appréhende » le futur, de façon à le consumer, à le manipuler et à en disposer. Mais en scrutant le futur, la possibilité de la mort apparaît, la possibilité de quelque chose ne pouvant être dominé. Cette dimension menaçante du futur peut mener encore une fois l'individu coupable dans une fantaisie ou une conception trompeuse de lui-même ; soit en se réfugiant de nouveau dans la sécurité du passé ou en se concentrant aveuglément sur le futur comme si la mort allait au dernier moment se dérober. Accablé par le poids du passé ou en le « revivant » de façon fantaisiste, appréhendant avaricieusement le futur ou effrayé par son incertitude, tous ces exemples de 
temporalité de la culpabilité ressemblent aux notions, d'« attenteoubli-présentifier », utilisées dans Être et Temps pour rendre compte de la forme inauthentique de la temporalisation.

Au contraire, la temporalité de la fidélité est reconnue par Heidegger comme le temps du kairos. A partir de l'analyse de certains verbes très présents ${ }^{43}$ dans les Lettres de Paul, Heidegger révèle qu'un chrétien fidèle découvre ce qui a été recouvert par la culpabilité et arrive ainsi à une compréhension de ce qu'il a toujours été : un être appartenant à Dieu. Cette découverte de ce qu'il a toujours été permet à l'homme d'aborder le futur avec une ouverture à la présence divine qui a toujours été présente. Cette présence n'est pas, comme nous le rappelle Zimmerman, la présence de Dieu commme objet dans le temps mais plutôt Dieu présent comme absent, comme la temporalité elle-même ${ }^{44}$. Le «Kairos » est la réalisation actuelle de ce qu'on a toujours déjà été et de ce qu'on sera : un être de temporalité finie. L'attente pour la parousia n'est donc pas l'appréhension d'un évènement unique pouvant être situé sur une continuité linéaire mais plutôt le partage fini du Dasein de la «temporalité primordiale et infinie » de Dieu $^{45}$.

Cette compréhension de la temporalité de l'authenticité comme dé-mythologisation de ce que Heidegger considère comme la temporalité de la fidélité nous permet maintenant d'aborder dans une autre perspective la question du rôle actif attribué au Dasein dans l'« accomplissement » de l'authenticité, et conséquemment, dans le dépassement de la « crise ». Comment advient exactement la fidélité ? Comment Heidegger conçoit-il l'accomplissement de la compréhension du temps (kairologique) accompagnant la fidélité ? Là encore, Heidegger est ambivalent, ce qui n'est pas surprenant. Il y a toujours eu, entre les différentes conceptions théologiques concrètes de la fidélité, une certaine tension à l'égard du rôle du soi dans la fidélité. Dans les termes les plus généraux, nous pourrions dire qu'il y a opposition entre une vision « catholique » et une version « protestante » de la fidélité, ce qui nous mène à une discussion portant, d'une part, sur la

43. Sheehan, « Heidegger's Introduction to Phenomenology of Religion », op. cit, p. 320-21.

44. Zimmerman, op. cit., p.r37.

45. Heidegger sur la possibilité de comprendre philosophiquement l'« éternité » de Dieu; voir sa note dans ÊT. p. 494. 
« grâce » et, d'autre part, sur les « bonnes œuvres ». Il n'est pas surprenant de retrouver des éléments constitutifs de ces deux points de vue dans les travaux du jeune Heidegger, qui était luimême un penseur formé dans le " Fribourg archi-catholique 》 (telle que décrite par Husserl dans une lettre à Rudolf Otto ${ }^{46}$, dont l'intérêt marqué pour Augustin, Luther et Kierkegaard le mena à de riches échanges avec des théologiens protestants tels que Bultmann et Paul Tillich. Alors que nous pouvons « uniquement être sauvé par la grâce », l'acceptation de cette grâce est à un certain point dépendante des dispositions de celui qui la reçoit. L'authenticité / fidélité signifie ultimement être vrai par rapport à soi-même, ou dans le langage des Lettres de Paul, être « obéissant » ${ }^{47}$. Et l'obéissance signifie « écouter ». Il est vrai que celui qui écoute n'a pas le plein contrôle sur ce qu'il écoute, mais il est également vrai qu'écouter n'est pas une expérience simple et purement passive. Chez le jeune Heidegger, nous retrouvons une très forte conviction que l'on se doit de prendre la décision d'écouter, d'être « obéissant » à son soi véritable. De ce point de vue, l'authenticité semble inhérente au contrôle du Dasein.

Nous nous devons de conclure qu'il y a certains aspects contradictoires dans la notion d'authenticité telle que Heidegger la pense. D'une part, aucune « activité » du Dasein ne sera ellemême suffisante pour accomplir l'authenticité, aucune activité ne permettra au Dasein de se découvrir lui-même. D'autre part, Heidegger croit, comme Husserl, qu'il est nécessaire de combattre pour obtenir cette découverte de soi, que l'on doit se battre pour se libérer des tendances du « on », et que nous devons constamment nous résoudre à demeurer disponible pour les possibilités authentiques qui, à la fin, sont donnèes au Dasein par le Dasein luimême. Heidegger tenta ensuite d'atténuer cette tendance volontariste présente dans ses premiers écrits. Dans L'origine de l'œuvre d'art datant de 1935-1936, il écrit :

L'ouverture pour la décision résolue, dans Sein und Zeit, n'est pas l'action décidée d'un sujet, mais l'ouverture du Dasein hors de l'engagement dans l'étant, jusqu'à l'ouvert de l'être. l...l Ni pour la création déjà nommée, ni pour le vouloir cité à présent, nous ne pensons à

46. Archives Husserl, R I Otto, 5.III. IgI9.

47. Le lien étymologique entre obedire / audire est maintenue en Allemand et en Néerlandais : gehoorzaamheid / horen ; Gehorsamkeit / hören. 
l'effort et à l'action d'un sujet se posant lui-même comme but et fin de toute reussite à laquelle il peut aspirer ${ }^{48}$.

Ce texte est éclairant pour une foule de raisons. Premièrement, il nous donne un autre exemple concret d'un « type » d'authenticité comprenant une interaction actif-passif essentielle : l'acte authentique de la création d'un artiste. Ici aussi, beaucoup d'aspects semblent être redevables à la donation : le talent, l'inspiration, les outils, l'entraînement. Par contre, l'artiste vraiment créatif n'est pas celui qui se contente de « répéter »ce qui a été transmis, mais celui qui le transforme en quelque chose d'autre, qui ouvre un espace pour une nouvelle révélation de l'être, qui combat avec les matériaux disponibles, qui tente de « dire » quelque chose et ce, non pas pour son propre compte mais pour le compte de ce qui doit être dit.

Ce texte est également éclairant car il advient au sein d'une discussion portant sur la connaissance et la volonté. Heidegger nous fait remarquer que si la production artistique comporte un acte de volonté, il ne s'agit pas de la volonté rationnelle, comme « la simple connaissance et représentation de quelque chose ${ }^{49}$. En effet, l'étude de l'interaction passif-actif au sein de l'authenticité nous fait remarquer la séparation instaurée entre la pensée heideggerienne et les schèmes traditionnels habituellement utilisés pour aborder les questions de la volonté, de la raison et de l'affectivité. Selon Heidegger, la passivité qui sous-tend l'authenticité n'est pas purement sensible. En même temps, la « décision » portant à répondre à l'appel de la fidélité ou de la muse artistique n'est pas une décision rationnelle. Le temps authentique de la fidélité, le kairos, qui correspond à la notion heideggerienne d'instant, n'est pas un temps durant lequel on prend une décision rationnelle et volontaire. Même si l'authenticité est redevable d'une « vision » ou d'une compréhension, il ne s'agit pas fondamentalement d'une compréhension intellectuelle. La décision jouant un rôle pour l'authenticité n'est pas basée sur la pensée qui calcule ou sur la «prudence ».

Même si il est vrai, pour Heidegger, que fides quarens intellectum, la compréhension n'est pas un élément essentiel à la vie authentique de la fidélité. De façon similaire, nous n'avons pas

48. « L'origine de l'œuvre d'art » dans Chemins qui ne mènent nulle part, Paris, Gallimard, p. $75-76$.

49. Ibid. 
besoin d'une compréhension intellectuelle de ce que l'on crée pour être un artiste. Nous nous devons de rajouter cependant que l'authenticité n'est pas réservée, selon Heidegger, à la connaissance théorique ni à aucune forme de comportement pratique ${ }^{50}$. Il n'y a aucune façon définie, aucun type de comportement nous permettant de dire que « cela est authentique ». Il n'y a aucune règle formelle nous permettant d'être un fermier, un artiste ou un croyant authentique.

Malgré le fait que la notion d'authenticité soit chez Heidegger plutôt indéterminée ${ }^{51}$, elle semble être un mode d'existence accessible à n'importe quel Dasein, qu'il soit occupé à quelque tâche que ce soit. Par contre, le modèle « artistique » de la vie authentique entraîne certaines tensions à l'intérieur de cette conception, supposément égalitaire, de l'authenticité. La fidélité est certainement un don que tous peuvent recevoir. Par contre, nous ne sommes pas tous des artistes. Cette situation paradigmatique peut être vue comme un autre exemple du combat que livre Heidegger au rôle redevable du « sujet » en ce qui concerne l'authenticité. Cette situation pourrait certes être redevable du « tournant » qu'effectue Heidegger de I920-2I à I936 par rapport à la question du volontarisme, changement qui comporte essentiellement deux aspects. D'une certaine façon, Heidegger développe une conception de l'authenticité moins égalitariste ; l'authenticité advient à travers l'artiste, le poète, le penseur, celui qui crée et qui représente une nouvelle possibilité pour la révélation de l'être. Par contre, il est vrai que ces quelques « élus » sont réellement choisis, mais ils sont ainsi réduits à être des voies pour la révélation de l'être lui-même.

Heidegger dépasse le volontarisme de ses débuts en affirmant ultérieurement que l'authenticité ne dépend pas tant de l'activité réceptive du Dasein que du don de l'être, du es gibt ${ }^{52}$. Dit autrement, Heidegger passe de la temporalité du Dasein à la manière dont le Dasein est possédé par la temporalité elle-même, ou d'un Dasein

50. $\hat{E} T$, P. $358-359$

51. La nature indéfinie de l'authenticité fut réduite à une blague par un des étudiants de Heidegger : «I Ich bin entschlossen, nur weiss ich nicht wozu ». Voir Löwith, Mein Leben in Deutschland vor und nach 1933. Ein Bericht, Stuttgart, J.B. Metzler, 1986, p. 29.

52. Martin Heidegger, « Zeit und Sein », dans Zur Sache des Denkens, Tübingen, Max Niemeyer Verlag, 1969 , p. 5 sq. 
comme temporalité finie ouverte à l'être comme avènement temporel [Ereignis].

Beaucoup de choses se produisent dans la pensée heideggerienne au cours de ce passage de la résolution authentique à la Gelassenheit. Comme le remarque ironiquement Derrida, en faisant référence à la période $1927^{-1953}$, ces années n'avaient rien d'ordinaire ${ }^{53}$. Les auto-interprétations de Heidegger sont intéressantes autant pour ce qu'elles révèlent que pour ce qu'elles cachent. Le dernier Heidegger souhaite clairement atténuer les tendances volontaristes retrouvées dans ses premiers travaux. Nous pourrions croire que cette situation est liée à son expérience comme Recteur en I933 et à la croyance, dont il était la proie à cette époque, qu'il avait à jouer un rôle actif en dirigeant l'université vers l'authenticité. Quoiqu'il fut conscient d'un type «nécessaire »d'inauthenticité, il y eut une période où le dépassement volontaire de l'inauthenticité était le but visé par le « leader spirituel » péremptoire de l'université allemande. Que Heidegger puisse être aussi enclin à la prescription indique non seulement les restes dans sa pensée de ce qu'il nommerait la «tradition métaphysique » mais également le danger impliqué lorsque quiconque prend son « authenticité » trop au sérieux.

\section{Université McGill}

(Tradutt de i'anglais par J.-F. Campeau)

53. Jacques Derrida, De l'esprit: Heidegger et la question, Paris, Galilée, 1987, p. I2. 